\title{
Epidemiology of Trachoma in Health District of Fria in 2014
}

\author{
Aly Sylla ${ }^{1,}$, , Seydou Bakayoko, Pierre Louis Lamah², Alexis Ouendouno², Amadou Sylla², \\ André Goepogui ${ }^{2}$, Rodrigue Romuald Elien Gagnan Yan-Zaou-Tou ${ }^{1, *}$, \\ Rahim Cheikh Nagnan Diabate ${ }^{1}$, Japhet Pobanou Thera ${ }^{1}$ \\ ${ }^{1}$ Institute of African Tropical Ophthalmology, University of Sciences and Technology of Bamako, Bamako, Mali \\ ${ }^{2}$ Faculty of Medicine, Gamal Adbel Nasser University of Conackry, Conackry, Guinea
}

Email address:

alysylla5316@gmail.com (A. Sylla), rodrigueelien@yahoo.fr (R. R. E. G. Yan-Zaou-Tou)

${ }^{*}$ Corresponding author

\section{To cite this article:}

Aly Sylla, Seydou Bakayoko, Pierre Louis Lamah, Alexis Ouendouno, Amadou Sylla, André Goepogui, Rodrigue Romuald Elien Gagnan Yan-Zaou-Tou, Rahim Cheikh Nagnan Diabate, Japhet Pobanou Thera. Epidemiology of Trachoma in Health District of Fria in 2014. Central African Journal of Public Health. Vol. 5, No. 6, 2019, pp. 261-265. doi: 10.11648/j.cajph.20190506.16

Received: September 17, 2019; Accepted: October 5, 2019; Published: October 16, 2019

\begin{abstract}
Introduction: Trachoma is a chronic keratoconjunctivitis caused by Chlamydia trachomatis bacteria. An epidemiological study was conducted in 2014 in the Health District of Fria (Northern Guinea province) to assess the prevalence of active and scar trachoma in a suspected endemic area of approximately 120-135 inhabitants. This is a transversal, descriptive, clustered survey of a representative sample of the population according to the standard protocol recommended by the World Health Organization (WHO). The clinical review of target populations was conducted according to WHO codification. Of the 3173 subjects recorded, 3159 subjects consented to be examined or an examination rate of $99,55 \%$. The prevalence of active trachoma was $8.67 \%$ (CI at $95 \%=7.2-10.4$ ). The prevalence of scar trachoma was $0.21 \%$. The principal sources of water for households are surface water (41\%) and drilling water (41\%). The prevalence of active and scar trachoma, risk factors are discussed. In our study, trachoma appeared as a public health problem in the health district of Fria. The prevalence of active and scar trachoma is approaching WHO limit. Poor hygiene was the main risk factor. The implementation of the different components of the strategy SAFE in this locality is a necessity.
\end{abstract}

Keywords: Prevalence, Trachoma, Fria

\section{Introduction}

Trachoma is a chronic keratoconjunctivitis caused by Chlamydia trachomatis bacteria, human-to-human transmission via nasal, ocular secretions and most likely favoured by flies that are passive vectors [1].

It is a major cause of blindness worldwide and one of the leading causes of infectious and preventable blindness worldwide [1]. Trachoma is generally found in desert and semi-desert rural areas and in the world's most disadvantaged communities, where water scarcity makes poor hygiene and living conditions more difficult with a dry climate and dusty environment in developing countries [2].

According to the WHO, in 2012, nearly 2.2 million people were visually impaired, and 1.2 million were blind [3].

Trachoma remains a major public health problem, the second leading cause of blindness after cataracts in developing countries and the first cause of avoidable blindness in the world. [2, 3]

In Cameroon, the study in district of Kolofata in December 2006 showed a prevalence of $21 \%$ follicular trachoma and $5.2 \%$ intense follicular trachoma in children aged bewteen 1 to 9 years old and a prevalence of $3,4 \%$ trichiasis entropion. [4]

In Senegal, the 2003 study showed a prevalence of active trachoma of $10.8 \%$ and $1.2 \%$ of intense trachoma in children aged 0 to 10 years, those of entropion trichiasis and corneal opacities in women over 14 years of age were estimated to be $2.6 \%$ and $1.4 \%$ respectively. [5]

In Guinea, a survey carried out by the Organization for the Prevention of Blindness (OPC) in 2002 concerned only one region, that of Haute-Guinée, bordering Mali (where the National Trachoma Prevalence Survey in 1996-1997 estimated active trachoma prevalence rates among children 
under 10 years old at $34.9 \%$ and $2.5 \%$ for trichiasis among women aged 15 years old and elder). The prevalence of active trachoma in children was $32.3 \%$, very close to that of neighbouring Malian regions. The same was true for entropion-trichiasis $(2.7 \%)$ and central corneal opacities $(0.8 \%)$ in women over 14 years old. [6]

\section{Patients and Methods}

a) Context: The health district of FRIA located in the north of the capital Conakry and has population of approximately at about 120 - 135000 inhabitants or a density of 66 inhabitants per $\mathrm{km}^{2}$. It includes a reference public hospital of district.

b) Period of study: Our study took place from 02 March 2014 to 07 April 2014.

c) Type of study: This is a one-pass, cross-sectional, twolevel cluster random sampling study.

d) Study population: The study involved children aged 1 to 9 years old for the assessment of the prevalence of active trachoma (TF/TI) and subjects aged 15 years old and over for the assessment of the prevalence of trachomageal trichiasis.

e) Sampling Plan: Sampling was done using a two-tier cluster random survey:

1. First level of survey:

It lists the villages. 20 villages (clusters) were randomly sampled from the frame using the random number table.

2. Second level of survey:

In each of the selected villages, a subsample of 30 households was drawn in which all persons aged one (1) year and over were examined.

For the random selection of the thirty (30) households, a list was made of all the names of the heads of households in the village who were assigned the order numbers. Of these numbers, thirty (30) were drawn and these thirty (30) households were the sample a listing was made of all the names of the heads of household in the village to whom the order numbers were assigned. Of these numbers, thirty (30) were drawn and these thirty (30) households formed the representative sample of the village.

According to the survey protocol, a woman, her children and the husband were treated as housekeeping, along with the patriarchs.

In polygamous families, lots were drawn to draw a single woman for examination, her children and her husband. These were the representative sample of the family.

f) Sample size:

She was represented by all persons residing in the household whose age was greater than or equal to one (1) year.

g) Survey team and materials:

It consisted of three (3) persons:

1. A clinical examiner for trachoma signs;

2. A recording agent (data entry operator) for data recording in the Smartphone;

3. A driver.
4. A guide (serving at the same time as an interpreter) was recruited from each village and made available to the investigators to accompany them in the selected households.

h) The technique of moving investigators from households:

Under the direction of the local guide, the itinerary was always prepared taking into account the geographical situation of the selected households, The crew started with the household furthest from the dealership where they were housed and ended with the nearest household to avoid unwanted movement.

In households where the absence was recorded, the team of investigators revisited these households at the end of the day to catch up with these absence cases, in order to examine and record them. Priority for this return was given to households where children aged one (1) to nine (9) were absent, as this age group is the primary target of trachoma.

i) The terms of the review:

This study included all consenting people, whether trachomateus or not, living in the selected households.

All cases of trachoma were diagnosed and recorded using the simplified coding of the WHO trachoma.

All children, after parental consent, and consenting adults were examined by an Ophthalmological Technician (TSO) with a binocular magnifying glass of power 2.5 under adequate lighting (preferably daylight), from trachoma to its various stages.

Variables related to age, sex and environment were noted (water availability and availability of latrines used).

j) Examination Procedure:

The examination was carried out using a binocular magnifier ( $\mathrm{X}$ 2.5) and under adequate lighting (daylight preferably).

The examiner first looks for the presence of eyelashes deflected towards the globe (TT: an eyelash, at least rubbing the eyeball. evidence of recent depilation of deviated cilia (s) was considered as trichiasis, followed by corneal opacity (CO: obvious corneal opacity over pupillary area), then it turns the upper eyelid and looks on the tarsal surface of the upper conjunctiva for signs of inflammation (TF: presence of 5 or more follicles on the upper tarsal conjunctiva, and TI: pronounced inflammatory thickening of the tarsal conjunctiva masking more than half of the deep vessels of the tarse) and scarring (TS: scarring on upper tarsal conjunctiva). Each eye is examined separately and any signs must be clearly seen to be present. In case of doubt, the sign is considered to be absent.

The adult subjects were seated in front of the examiner, who himself was seated.

The children could stand in front of the examiner. As for very young children, their heads were placed between the examiner's knees with their faces facing up, the child's body being firmly held on the knees of another adult (preferably the mother or guardian of the child) sitting in front of the examiner.

Examiner cleaned hands after each examination with 
appropriate disinfectant (Dakin or alcohol) or washed hands with water and soap.

He also ensured that all data collected in the smartphone was recorded.

People with signs of active trachoma were treated with $1 \%$ tetracycline ophthalmic ointment. Subjects with trachomageal trichiasis were referred to specialist centres for treatment.

k) Ethical considerations:

To complete this study, we were selected from the ORLO Chair to PNLOC as part of the finalization of trachoma mapping in Guinea where we had received training prior to being in the field.

In addition, the activities were explained to local and regional authorities with a view to obtaining their membership and authorisation by presenting them with the order of mission issued from the Guinea Ministry of Health.

At the community level, the purpose of the survey was explained to village heads, followed by heads of households and individuals. In the case of children, this explanation was given to the parents or their guardians. A verbal agreement was given by the heads of villages, households or guardians. Subjects were informed that they have the right to participate or not in the study without losing any future interests of the
PNLOC/MTN. There was no premium for participation in the study. Information on the status of each trachoma-related subject was confidential. However, anyone diagnosed with active trachoma received two (2) tubes of $1 \%$ Tetracycline ophthalmic ointment. Subjects with trachomal trichiasis (TT) were referred to the nearest sanitary structure and the most suitable for the treatment.

1) Data entry and analysis:

The GTMP "Links system" made it possible to develop the questionnaire, collect and clear the database on an android platform using Motorola smartphones.

The data analysis was done using Epi-infos 6.0 software.

The graphs were made by Microsoft Excel 2007 software.

\section{Results}

In the district of Fria, we recorded 3,173 subjects divided among 480 households.

Of the 3,173 subjects recorded, 3,159 subjects consented to be examined and 14 subjects were absent.

Prevalence of active trachoma $(\mathrm{TF} / \mathrm{TI})$ in children aged bewteen 1-9 years old.

Out of 1,505 children aged 0-9 years old examined 130 had active trachoma (TF/TI) or 8.67\% (95\% CI: 7.2-10.4).

Table 1. Distribution of active trachoma by age of children.

\begin{tabular}{lllll}
\hline \multirow{2}{*}{ Age (Years old) } & Sample & \multicolumn{3}{c}{ Active Trachoma (TF/TI) } \\
\cline { 2 - 5 } & Frequency & \% & Frequency & \% \\
\hline 1 & 115 & 7.6 & 8 & 6.15 \\
2 & 116 & 7.7 & 12 & 13.08 \\
3 & 183 & 12.2 & 17 & 11.54 \\
4 & 181 & 12. & 15 & 17.69 \\
5 & 203 & 13.5 & 23 & 15.38 \\
6 & 182 & 12.1 & 20 & 9.23 \\
7 & 188 & 12.5 & 12 & 5.38 \\
8 & 136 & 9. & 7 & 12.31 \\
9 & 201 & 13.4 & 16 & 100 \\
\hline
\end{tabular}

Children aged 5 and 6 years old were the most affected with prevalence rates of $17.69 \%$ and $15.38 \%$ respectively.

Table 2. Distribution of Active Trachoma (TF/TI) by Primary Source of Drinking Water for Households during the Dry Season.

\begin{tabular}{|c|c|c|c|c|}
\hline \multirow{2}{*}{ Water source } & \multicolumn{2}{|l|}{ Courtyards } & \multicolumn{2}{|c|}{ Active trachoma } \\
\hline & Frequency & $\%$ & Frequency & $\%$ \\
\hline Surface water (e.g. rivers, dams) & 228 & 48 & 53 & 41 \\
\hline Tubed well or drilling & 118 & 25 & 53 & 41 \\
\hline A protected dug well & 48 & 10 & 9 & 7 \\
\hline Tap water at home & 0 & 0 & 7 & 5 \\
\hline Unprotected source & 60 & 13 & 0 & 0 \\
\hline Tap water in the courtyard & 10 & 2 & 0 & 0 \\
\hline Public tap water & 0 & 0 & 0 & 0 \\
\hline Protected source & 0 & 0 & & \\
\hline Unprotected dug well & 16 & 3 & 8 & 6 \\
\hline Water for sale & 0 & 0 & 0 & 0 \\
\hline Rainwater collection & 0 & 0 & 0 & 0 \\
\hline Total & 480 & 100 & 130 & 100 \\
\hline
\end{tabular}

The principal sources of water for households are surface water (41\%) and drilling water (41\%). 
Table 3. Distribution of the prevalence of active trachoma by type of latrine in courtyeards.

\begin{tabular}{llll}
\hline \multirow{2}{*}{ Latrines } & Courtyeards & \multicolumn{2}{c}{ Active trachoma } \\
\cline { 2 - 4 } & Frequency & \% & Frequency \\
\hline No toilet or scrubland or farm & 254 & 53 & 80 \\
Slab/open pit latrines & 167 & 35 & 62 \\
Pit latrines with slab & 55 & 11 & 0 \\
Improved Ventilated Pit Latrines (IVP) & 4 & 1 & 37 \\
Total & 480 & 100 & 13 \\
\hline
\end{tabular}

More than half of our subjects (62\%) were defecating in the scrubland.

Table 4. Active Trachoma (TF/TI) and Presence of Fly on Child's Face.

\begin{tabular}{lllll}
\hline \multirow{2}{*}{ Flies } & Sample & & Active trachoma (TF/TI) \\
\cline { 2 - 5 } & Frequency & \% & Frequency \\
\hline Présence & 49 & 10,2 & 27 & \% \\
Absence & 431 & 89,8 & 103 & 21,43 \\
Total & 480 & 100 & 130 & 27,08 \\
\hline
\end{tabular}

$\mathrm{X}^{2}=10,462 \mathrm{df}=1 \mathrm{P}<5 \%$.

The presence of flies on the face at the time of the examination showed a prevalence of $5.63 \%$ active trachoma.

Table 5. Distribution of trichiasis trachoma prevalence among adults aged 15 year old and over by age group.

\begin{tabular}{|c|c|c|c|c|}
\hline \multirow{2}{*}{ Age group (Years old) } & \multicolumn{2}{|l|}{ Sample } & \multicolumn{2}{|c|}{ Trichiasis Trachoma } \\
\hline & Frequency & $\%$ & Frequency & $\%$ \\
\hline $15-24$ & 250 & 17.83 & 0 & 0 \\
\hline $25-34$ & 275 & 19.61 & 0 & 0 \\
\hline $35-44$ & 311 & 22.18 & 0 & 0 \\
\hline $45-54$ & 228 & 16.26 & 1 & 33.33 \\
\hline $55-64$ & 166 & 11.84 & 2 & 66.67 \\
\hline $65-74$ & 131 & 9.34 & 0 & 0 \\
\hline 75 and more & 41 & 2.92 & 0 & 0 \\
\hline Total & 1402 & 100 & 3 & 100 \\
\hline
\end{tabular}

\section{Discussion}

\subsection{Result on the Trachome}

The prevalence of active trachoma at FRIA was $8.64 \%$ (among children 1-9 years old).

TF prevalence was $8.50 \%$ and IT was $1 \%$. Children aged 5 years old were more affected with a prevalence of $17.96 \%$ followed by children aged 6 years old with a prevalence of $15.63 \%$.

The prevalence of trachomateux trichiasis in subjects 15 years old and older was $0.21 \%$.

Our results are different from those found in Mali in 2015 with prevalence variants of $23 \%$ to $81 \%$ for active trachoma and $9 \%$ to $65 \%$ for trichiasis trachoma [7].

The smaller sample size would justify the difference in results.

\subsection{Risk Factors}

a) The sex:

Female children aged 1-9 years old were the most affected with a prevalence of $53.1 \%$. Our observation is identical to the authors' observations in Mali and Ethiopia [7, 8]. The explanation comes from the fact that the female sex, even at a young age, would be more exposed to trachoma than the male sex, due to the role played by women in African society

b) The age:

In our study we found only the high prevalence of active trachoma in 5-year-olds with $17.96 \%$ followed by 6 -yearolds with $15.63 \%$. The maximum prevalence found at 5 years old and 6 years old is well above the WHO threshold (< $10 \%$ ) emphasizes the importance of trachoma as a public health problem in Guinea on the one hand and would indicate a certain a breakdown of contact between the mother and the child on the other hand [9]. As a result, the mother pays very little attention to the child's personal hygiene and clothing, which exposes him to the risk of trachomateusis. Also the main games at the age of 5 and 6 years oldare often in the dust; this would explain the high risk of the occurrence of trachoma in this age group.

c) Trachoma and water:

In our study, active trachoma was most common in households that used either tubed wells $(41 \%)$ or surface water (41\%) As the main source of drinking water during the dry season, however, it was almost absent in households that used tubed wells or drilled wells, protected dug wells. This observation is supported by the literature [10] which states that trachoma occurs most frequently in communities or concessions where water supply is difficult. This difficulty in accessing water 
results in less use in rural areas in Africa. The risk of the disease is related to poor water management to keep children clean.

d) Trachoma and latrine:

Our study showed that trachoma was more common in children living in households without latrine $(61.5 \%)$ than in children living in households with ventilated pit improved latrine $(10 \%)$.

Our results are in line with those of Schemann et al. which showed that the presence of functional latrines in concessions or houses was associated with lower trachoma prevalences in several countries. [11]

e) Trachoma and flies on face:

The presence of flies on children's faces was significantly associated with active trachoma in $5.63 \%$ of cases. Our result is consistent with that of the study conducted by J. F. SCHEMMAN [12] in 2001-2002 in three countries of the sub-region showed a high prevalence when the face was dirty.

\section{Conclusion}

The objectives of this study were to assess the prevalence of active trachoma in children 1 to 9 years old, to assess the prevalence of trachoma trichiasis in adults 15 years old and elder, and to describe the main risk factors.

1505 children were examined for active trachomage (TF/TI). 1402 adults were examined for trachomageal trichiasis. The choice of a cross-sectional survey enabled us to carry out this study. In Fria, the prevalence of active trachoma in children aged 1 to 9 years was $8.50 \%$. Girls appeared to be the most affected than boys with $53.10 \%$ and $46.9 \%$ respectively the prevalence of trachomageal trichiasis in adults aged 15 years and over was $0.21 \%$ close to the $1 \%$ WHO threshold. Poor domestic hygiene promotes disease and increased trachoma has not been observed when latrines do not exist.

The availability of quality water would help to effectively control the condition but at the same time make the right use.

\section{References}

[1] Taylor HR, Burton MJ, Hadda D, West S, Wright H. Trachoma. Lancet, 2014; 384: 2142-2152.
[2] Anne-Marie MOULIN, Jeanne ORFILA, Doulaye SACKO, Jean-François SCHEMANN. Lutte contre le trachome en Afrique Sub saharienne. Ed. IRD, Paris 2006. 225 pp. ISSN 1633-9924/ISBN 2-7099-1601-0.

[3] World Health Organization. Rapport de situation sur l'élimination du trachome 2012. REH, 2013, 88 (24): 241256.

[4] P. Bensaid, P. Goldschmidt, P. Huguet, E. Einterz. Enquête de prévalence du trachome cécitant dans le district de Kolofata, nord Cameroun: déroulement pratique, résultats, perspectives de traitement. J. Fr. Ophtalmol. 2007, 30 (2): 2 S302. https://doi.org/10.1016/S0181-5512(07)80341-X.

[5] M. B. SAAL, J. F. SCHEMANN, B. SAAR, M. FAY E, G. MOMO, S. MARIOTTI, A. D. NEGREL. Le Trachome Au Senegal: Resultats d'une Enquete Nationale. Med Trop 2003; 63: 53-59.

[6] GeOPOGUI A., HUGUET P., MARIOTTI S., P., TRAORE L., GODIN-BENHAIM C., NEGREL A. D., Trachome en Guinée (Conakry). Rev. Int. Trach. 2002, 77-79: 151-153.

[7] Traoré L, Dembele B, Keita M, Reid SD, Dembele M, Mariko B, et al (2018) Prevalence of trachoma in the kayes region of Mali eight years after stopping mass drug administration. PLoS Negl Trop Dis 12 (2): e0006289.

[8] Ayana Tsega Ferede, Abel Fekadu Dadi, Amare Tariku and Akelew Awoke Adane. Prevalence and determinants of active trachoma among preschool-aged children in Dembia District, Northwest Ethiopia. Infectious Diseases of Poverty, 2017, 6: 128.

[9] Victor H. HU, Emma M. Harding-Esch, Matthew J. Burton, Robin L. Bailey, Julbert Kadimpeul and David C. W. Mabey. Epidemiology and control of trachoma: systematic review. Tropical Medicine and International Health. 2010, 15 (6): 673691.

[10] SCHEMANN. J. F., SACKO D., MALVY D., BANOU A., TRAORE L., MOMO G., 2002. Risk factors for trachoma. Int. J. Epidemiol, 31: 194-201.

[11] SCHEMANN JEAN FRANÇOIS. «Le trachome, une maladie de la pauvreté», éd. IRD, Paris, 2008, 206 pp. ISBN: 978-27099-1646-2/ISSN: 1142-2580.

[12] SCHEMANN. J. F. Trachome et xerophtalmie en Afrique; Deux maladies de société. Thèse sciences biol Med, Université victor Segalen, Bordeaux II. 2002. 